\title{
IMPROVING PRESCRIBING FOR PAIN: GUIDELINES FOR GENERAL PRACTITIONERS PRESCRIBING FOR MIGRAINE AND LOW BACK PAIN
}

Karen Kaye and Wendy Rotem

NSW Therapeutic Assessment Group

This report describes two new guidelines for general practitioners (GPs), aimed at improving prescribing for pain associated with migraine and low back pain, which have been distributed recently in NSW. Both guidelines were prepared by the NSW Therapeutic Assessment Group (NSW TAG), with a grant from the NSW Department of Health, in response to a need for better information on the prescribing of opioids. NSW TAG is a committee of clinical pharmacologists, pharmacists and clinicians who are committed to promoting the quality use of medicines in hospitals and in the wider community. The guidelines integrate clinically appraised evidence from the medical literature with knowledge gained from relevant clinical experience. The unique feature of these guidelines is that they were prepared in consultation with GPs, with their needs specifically in mind. A complementary education strategy has also been implemented.

\section{BACKGROUND}

Pain has been identified as one of the three most costly areas of health care in Australia. Both undertreatment and overtreatment of pain are associated with disability, suffering and escalating cost. Therefore appropriate treatment of pain, especially non-malignant pain (both acute and chronic), is a subject of concern to prescribers, funders and consumers.

Among the many painful conditions that lead people to consult their GPs, migraine and back pain are two of the most prevalent. Migraine, for example, occurs in 10 to 20 per cent of the population and has been reported to account for approximately one in every 140 occasions of service in general practice. Similarly low back pain is reported to be one of the most prevalent health problems in the general population.

Despite the advent of new therapies, these common conditions can be complex and difficult to treat. In some cases doctors and patients resort to opioid analgesics such as pethidine in an effort to achieve pain relief. Unfortunately patients can become dependent on these drugs, requiring escalating doses (with an accompanying increase in dysfunction) while the underlying problem is not resolved.

There is evidence of an increase in opioid prescribing for non-malignant pain associated with these conditions. In this context the Pharmaceutical Services Branch of the NSW Department of Health commissioned this project because of concern about the number of prescriptions for pethidine, and the potential for the associated problems of addiction and drug-seeking behaviour.

\section{PREPARATION OF THE GUIDELINES}

The aims of this project were to:

- improve the selection of analgesics by GPs managing migraine and low back pain;

- reduce the prescription of pethidine for these conditions;

- raise community awareness about appropriate treatment of migraine and low back pain.

An 11-member committee was established to guide the project. It consisted of representatives from the Royal Australian College of General Practitioners (RACGP), NSW Divisions of General Practice, NSW Health Pharmaceutical Services Branch, drug and alcohol specialists, neurologists, rheumatologists, and consumers. A review of current evidence and existing guidelines provided the basis for initial drafts of each guideline, which were distributed to 10 Divisions of General Practice for written comment on the content, format, applicability in general practice, and educational support requirements. A subsequent draft was sent for comment to all NSW Divisions of General Practice, NSW Department of Health, RACGP and other relevant colleges and organisations, consumer organisations and other interested parties.

The resulting guidelines for migraine recommend that the most effective treatment is aspirin or paracetamol commenced as soon as possible after the onset of the attack - usually at the onset of the migraine aura-plus an anti-emetic if vomiting accompanies the attack. For low back pain, paracetamol or a non-steroidal antiinflammatory drug, and a graded exercise program, is the recommended treatment. Pethidine's short duration of action generally provides little benefit in controlling the prolonged and recurring pain associated with these conditions. If stronger analgesics are required then longacting oral forms are preferable to short-acting injectable forms like pethidine.

The final guidelines were printed in a format that had been identified during the consultation process as userfriendly for GPs. The guidelines were launched in January 1999 by the NSW Minister for Health, and 10,000 copies were distributed to all GPs in NSW and to relevant organisations and individuals. The guidelines are also available in electronic version via the Internet at www.medeserv.com.au/tag.

\section{EDUCATION STRATEGIES}

Feedback from GPs and others indicated that patient education was an important aspect of the successful management of migraine and low back pain. Consequently patient brochures were developed, with input from patient support groups, to complement the guidelines. Eighty 
thousand brochures were distributed to GPs and patient support groups.

A workshop was provided for staff from the Pharmaceutical Services Branch of the NSW Department of Health, which focused on the management of pain and the use of the guidelines. The goal of the workshop was to facilitate the implementation of the guidelines by staff who are involved with advising doctors with regard to the prescription of opioids. Liaison with Divisions of General Practice was undertaken to encourage inclusion of the guidelines in their continuing education programs.

\section{CONCLUSION}

NSW TAG's guidelines for general practitioners prescribing for migraine and low back pain have been very positively received by both general practitioners and consumers. They provide recommendations based on the principles of evidence-based medicine and incorporate clinical experience and accepted practice.
An additional grant from the NSW Department of Health is currently facilitating the formal evaluation of outcomes from use of the guidelines in several Divisions of General Practice. it

Both guidelines, and their companion patient information brochures, can be accessed via the Internet at www.medeserv.com.au/tag. Copies of the guidelines (but not the patient brochures) are available from the Better Health Centre. Phone (02) 98160353 and quote publication numbers PSB 990134 for migraine and PSB 990135 for lower back pain. Further information can be obtained from NSW TAG by phone (02) 93612852 , or by email at nswtag@stvincents.com.au.

\section{NSW AREA HEALTH SERVICE HEALTH STATUS PROFILES}

\section{Michele Puech and Helen Moore}

Population Health Indicators Unit

Epidemiology and Surveillance Branch

NSW Health Department

This article reports on the NSW Area Health Service Health Status Profiles, which have been produced as companion documents to each of the Performance Agreements (PAs) for the 17 Area Health Services (AHSs) in NSW.

\section{BACKGROUND AND PURPOSE}

The NSW Area Health Service Health Status Profiles are on-going surveillance reports on a set of key population health status indicators for each of the AHSs. The Profiles are produced by the Epidemiology and Surveillance Branch in consultation with relevant groups within the NSW Department of Health and the AHSs. The initial purpose of the Profiles as companion documents to the 1999-2001 AHS PAs was to provide:

- summaries of each AHS's standing in relation to key statewide health issues as a context for the negotiation of PAs at the area level;

- background population health data for annual AHS performance reviews;
- summaries of the health status of the populations of particular AHSs in relation to the state and averages for urban and rural areas.

The Profiles will be updated annually and published electronically.

\section{CONTENT}

The content of the Profiles was agreed upon by the NSW AHS Performance Agreement Steering Committee. The first edition, 1999-2000 NSW Area Health Service Health Status Profiles, is based on the indicators included in The Health of the People of NSW: Report of the Chief Health Officer (1997), the 1997 NSW Health Survey Electronic Report, and the AHS profiles used for the most recent round of performance reviews between the DirectorGeneral and AHS Boards. While the initial set of indicators was kept to a minimum, they still provide a reasonably comprehensive picture of the demographics and health status of the population of an AHS. For later editions the indicators will be reviewed and refined through further consultation with various content experts and AHS representatives.

The profile for each AHS includes sections on:

- demographics

- overall health status 